REPLY

\section{The complement system is also important in immunogenic cell death}

\author{
Lorenzo Galluzzi, Aitziber Buqué, Oliver Kepp, Laurence Zitvogel \\ and Guido Kroemer
}

We have recently reviewed current knowledge on the mechanisms that underlie immunogenic cell death (ICD) in the context of cancer and infectious diseases, the capacity of ICD to elicit an adaptive immune response in the absence of exogenous adjuvants, and the pathophysiological relevance of this process (Nat. Rev. Immunol. doi: 10.1038/nri.2016.107 (2016) $)^{1}$.

In their Correspondence (Nat. Rev. Immunol. doi: 10.1038/nri.2016.142 (2016)), Jin and He evoked the importance of the complement system for ICD, pointing to the role of complement activation in the efficient uptake of dead cells by phagocytes coupled to the delivery of robust anti-inflammatory signals. Activation of the complement system has indeed been linked to the capacity of phagocytes to engulf dead cells, while preserving tolerance ${ }^{2}$. Moreover, defects in the complement system in humans are associated with autoimmune disorders such as systemic lupus erythematosus (SLE) ${ }^{3}$. However, C3-deficient mice (which lack a convertase involved in both classical and alternative complement activation $)^{4}$ are viable and fertile, do not spontaneously develop autoimmune disorders, and only display mildly impaired B cell and $\mathrm{T}$ cell responses ${ }^{5,6}$. These findings suggest that systems other than complement have an even more important role in the maintenance of peripheral tolerance to dying cells ${ }^{7}$. Consistent with this interpretation, $\operatorname{Jmj} \mathrm{C}^{-1-}$ mice (which lack the phagocytic receptor for phosphatidylserine externalized by dying cells) die at birth owing to the accumulation of dead cells in the brain and lung ${ }^{8,9}$. Along similar lines, $\mathrm{Rubcn}^{-1-}$ mice (which are deficient in LC3-associated phagocytosis - a specialized phagocytic pathway relying on elements of the autophagic machinery $)^{10,11}$ spontaneously develop an SLE-like disorder owing to an intrinsic defect in efferocytosis ${ }^{12}$. Taken together, these observations point to complement activation as a process that supports, but is not crucial for, the removal of dying cells in physiological and pathological conditions. Irrespectively, our Review has a specific focus on the mechanisms of immunogenic, not tolerogenic, cell death.

Lorenzo Galluzzi is at the Department of Radiation Oncology, Weill Cornell Medical College, $10065 \mathrm{New}$ York, New York, USA; Equipe 11 Labellisée Ligue contre le Cancer, Centre de Recherche des Cordeliers, 75006 Paris, France; INSERM, U1138, 75006 Paris, France; Université Pierre et Marie Curie/Paris VI, 75006 Paris,

France; and Gustave Roussy Comprehensive Cancer Institute, 94805 Villejuif, France.

Aitziber Buqué is at the Equipe 11 Labellisée Ligue contre le Cancer, Centre de Recherche des Cordeliers, 75006 Paris, France; INSERM, U1138, 75006 Paris, France; Université Pierre et Marie Curie/Paris VI, 75006 Paris, France; and Gustave Roussy Comprehensive Cancer Institute, 94805 Villejuif, France.

Oliver Kepp is at the Equipe 11 Labellisée Ligue contre le Cancer, Centre de Recherche des Cordeliers, 75006 Paris, France; INSERM, U1138, 75006 Paris, France; Université Pierre et Marie Curie/Paris VI, 75006 Paris, France; and Metabolomics and Cell Biology Platforms, Gustave Roussy Comprehensive Cancer Institute, 94805 Villejuif, France.

Laurence Zitvogel is at the Gustave Roussy INSERM, U1015, 94805 Villejuif, France; Center of Clinical Investigations in Biotherapies of Cancer (CICBT) 1428, 94805 Villejuif, France; and Université Paris Sud/ Paris XI, 94270 Le Kremlin-Bicêtre, France. Comprehensive Cancer Institute, 94805 Villejuif, France;
Guido Kroemeris at the Equipe 11 Labellisée Ligue contre le Cancer, Centre de Recherche des Cordeliers, 75006 Paris, France; INSERM, U1138, 75006 Paris,

France; Université Pierre et Marie Curie/Paris VI, 75006 Paris, France; Metabolomics and Cell Biology

Platforms, Gustave Roussy Comprehensive Cancer Institute, 94805 Villejuif, France; Karolinska Institute, Department of Women's and Children's Health, Karolinska University Hospital, 17176 Stockholm, Sweden; and Pôle de Biologie, Hopitâl Européen George Pompidou, AP-HP; 75015 Paris, France

Correspondence to L.G. and G.K. deadoc@vodafone.it; kroemer@orange.fr doi: $10.1038 /$ nri.2016.143 Published online 28 Dec 2016

1. Galluzzi, L., Buqué, A., Kepp, O., Zitvogel, L. \& Kroemer, G. Immunogenic cell death in cancer and infectious disease. Nat. Rev. Immunol. http://dx.doi.org/10.1038/nri.2016.107 (2016).

2. Ogden, C. A. et al. C1q and mannose binding lectin engagement of cell surface calreticulin and CD91 initiates macropinocytosis and uptake of apoptotic cells. J. Exp. Med. 194, 781-795 (2001).

3. Sturfelt, G. \& Truedsson, L. Complement in the immunopathogenesis of rheumatic disease. Nat. Rev. Rheumatol. 8, 458-468 (2012).

4. Morgan, B. P. \& Harris, C. L. Complement, a target for therapy in inflammatory and degenerative diseases. Nat. Rev. Drug Discov. 14, 857-877 (2015).

5. Dempsey, P. W., Allison, M. E., Akkaraju, S., Goodnow, C. C. \& Fearon, D. T. C3d of complement as a molecular adjuvant: bridging innate and acquired immunity. Science 271, 348-350 (1996).

6. Kopf, M., Abel, B., Gallimore, A., Carroll, M. \& Bachmann, M. F. Complement component C3 promotes T-cell priming and lung migration to control acute influenza virus infection. Nat. Med. 8 373-378 (2002)

7. Galluzzi, L., Buque, A., Kepp, O., Zitvogel, L. \& Kroemer, G. Immunological effects of conventional chemotherapy and targeted anticancer agents. Cancer Cell 28, 690-714 (2015).

8. Fadok, V. A. et al. A receptor for phosphatidylserinespecific clearance of apoptotic cells. Nature 405, 85-90 (2000).

9. Li, M. O., Sarkisian, M. R., Mehal, W. Z., Rakic, P. \& Flavell, R. A. Phosphatidylserine receptor is required for clearance of apoptotic cells. Science 302 1560-1563 (2003).

10. Sica, V. et al. Organelle-specific initiation of autophagy Mol. Cell 59, 522-539 (2015).

11. Martinez, J. et al. Molecular characterization of LC3-associated phagocytosis reveals distinct roles for Rubicon, NOX2 and autophagy proteins. Nat. Cell Biol. 17, 893-906 (2015).

12. Martinez, J. et al. Noncanonical autophagy inhibits the autoinflammatory, lupus-like response to dying cells. Nature 533, 115-119 (2016).

Competing interests statement

The authors declare no competing interests. 